\title{
Simultaneous Recording of Urethral Pressure and Cross Sectional Area Profile
}

\author{
Tadashi Harada, Teruaki Kigure, Osamu Nishizawa, Seigi \\ TSUCHIDA and Jiro WATARAI* \\ Department of Urology and *Radiology, Akita University School of Medicine, \\ Akita, Japan
}

\begin{abstract}
A new technique for simultaneously recording urethral closure pressure and cross sectional area profile is described. The cross sectional area of the urethra could be measured using the field gradient principle. The procedure was performed readily in male patients and the recordings can be graphically presented during measurement. A high degree of reproducibility using this method was obtained. Typical recordings of control subjects as well as patients with pathology were obtained using this technique. The results of this study show that the maximum urethral closure pressure obtained was $60.8+3.4$ $\mathrm{mmHg}$ and functional profile length, $4.8+0.4 \mathrm{~cm}$. Internal meatus cross sectional area was $0.67+0.04 \mathrm{~cm}^{2}$ and the minimum urethral cross sectional area was $0.12+0.02 \mathrm{~cm}^{2}$. This method has been shown to be readily applicable in measuring urethral closure pressure in males and can potentially be used as a tool of comparing the functional parameters of pressure with the anatomical value of diameter.
\end{abstract}

Key words: Urethral pressure profile, urethral cross sectional area, urethral closure pressure

\section{Introdution}

The measurement of urethral pressure constitutes an important part of the clinical investigation of the cause of obstruction and the mechanism of continence. Currently, there are three basic techniques for recording the urethral pressure profile: (1) perfusion, (2) membrane catheter, or (3) micro-tip transducer. The perfusion technique for recording urethral profile has been widely applied during urodynamic tests following the report of Brown and Wicham (1967). In most cases, the catheter is perfused with water, using a syringe pump at a fixed rate from a side hole or from the tip. Thus, perfusion methods register the resistance to flow of the exit point of the catheter. The advantage of this method is that it is simple to set up and be interpreted in hydrostatic terms. However, in the perfusion system, factors such as perfusate characteristics, water or gas, the perfusion rate, catheter designed axial position of the catheter in cases of side hole arrangement, can affect the pattern and reproducibility of urethral pressure profile (Brown et al., 1967 ; Shmit et al., 1976). In addition, this method is not suitable for accurately recording pressure changes (Asmussen et al., 1976), such as coughing or strictures. 
Furthermore, urethral pressures increase by increasing distention of the smooth muscle surrounding the structure (Regnier et al., 1983 ; Colstrup et al., 1983; Harada, 1985), may produce a physiological change in the mechanisms of closure in addition to irritation.

The principle of measurement of urethral closing pressure, using a membrane catheter reported by Simons (1936) as a sphincterometry, is based on the compression of a soft balloon by surrounding urethral structure. Enhorning (1961) was able to record the intravesical pressure and the intraurethral pressure simultaneously using a two-channel, fluid-filled catheter connected to a strain gauge transducer. Tanagho et al. (1967) reported that continuous withdrawal of the membrane catheter from the internal meatus to the outside permitted recording of pressure with in the urethral lumen at various levels, and was an accurate and reproducible method that could be compared to the perfusion profilometry.

The micro-tip transducer technique has been reported to be another accurate, reproducible method for recording urethral pressure and urethral pressure profile (Asmussen et al., 1976 ; van Green, 1984). However, the literature pertaining to the micro-tip transducer method is such that the pattern of urethral pressure profile is influenced greatly by the axial position of the catheter. Some catheters are also quite rigid and their manipulation for insertion in the male is difficult. For these reasons, other ways of measuring urethral pressure are sought. Recently, Colstrup et al. (1983) combined the two parameters of cross-sectional area and urethral pressure in the resting female urethra. This was done by using a specifically constructed catheter with an electrical means to measure cross sectional area. In previous publications, we have also reported the use of urethral pressure and cross-sectional area to compute urethral compliance in the dog (Harada, 1985) and also in the human male (Harada et al., 1989). Using data from these measurements to calculate urethral compliance provides more accurate and complete information on the physical properties of urethral parameters and reflects urethral function more accurately. However, these measurements are too complicated and time consuming for routine clinical use, since they require further analysis following data collection.

To simplify the procedure we designed a new approach for simultaneous profilometry of urethral pressure and cross-sectional area, which we report in this paper. Using this new technique, we were able to evaluate its reproducibility, and illustrate, with some typical recordings obtained from different patients, the amount of useful information that can be produced.

\section{Method}

The profilometry probe consists of a 9 Fr. triple-lumen polyurethane catheter with a cylindrical balloon made of very thin silastic rubber membrane $8 \mathrm{~cm}$ from the tip (Figure 1). One lumen of the catheter is open-ended with a side hole at the tip for cystometry. The second lumen is open-ended beneath a balloon to measure urethral pressure and cross-sectional area, and the third lumen is used for a pathway of electrical connections. Two pairs of generating and detecting electrodes are mounted inside the balloon to measure the cross-sectional area of the balloon. The cross-sectional area of the urethra can be measured by the field gradient principle, using an impedance plethysmograph amplifier (Nihon Koden AI-601G). In this way, 


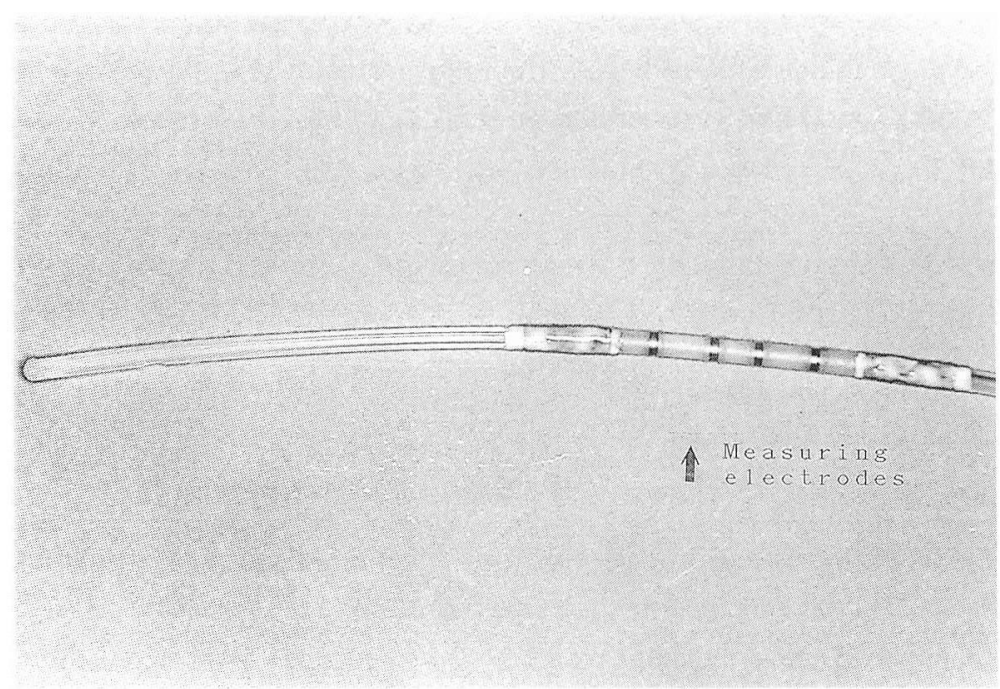

Fig. 1. Photograph of probe tip for simultaneous recording of urethral pressure and cross sectional area profile.

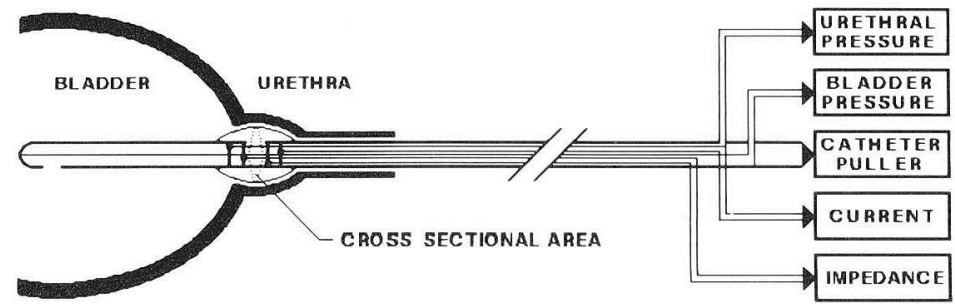

Fig. 2. Schematic diagram used for the simultaneous recording of urethral pressure and cross sectional area profile.

it is possible to simultaneously measure pressure of the bladder and the urethra, using a pressure transducer (Statham P-50) and a bridge amplifier (Nihon Koden AP-601G).

Urethral pressure profiles and cross-sectional area are obtained by gradually withdrawing the probe, using a catheter puller apparatus (Nihon Koden CW-201G) at a rate of $50 \mathrm{~mm} / \mathrm{min}$. Both urethral profilometries and urinary bladder pressure were recorded simultaneously by a polygraph system (Nihon Koden PR-3000). A schema of the instrumentation used to carry out the measurements is shown in Figure 2.

Simple profilometries are first obtained while the subject is at rest in the lithotomy position. The catheter is introduced into the bladder and the balloon of the catheter lining the bladder is inflated with saline until a $0.7 \mathrm{~cm}^{2}$ cross sectional area of the balloon is indicated. Then the value of balloon pressure is readjusted and set to equal the value of bladder pressure which is obtained through the other channel of the open-ended catheter located at the tip. The catheter is withdrawn mechanically at a rate of $50 \mathrm{~mm} / \mathrm{min}$ by a motor drive puller. The position of the balloon catheter was verified using a transabdominal ultrasound monitor (Yokokawa RT3000).

The reproducibility of this technique was examined in three healthy males between 33 and 
48 years of age. Three or four successive recordings of the profilometry were performed in each patient. The variables obtained from the measurements, i.e., maximum urethral closure pressure (UCPmax) ; functional urethral length (FUL); minimum urethral cross-sectional area (UCSAmin) ; and urethral cross-sectional area at the internal meatus (UCSAint) were presented separately.

In patients with voiding difficulty; stress urethral profilometry obtained as a response to coughing, voluntary contraction or electrical field stimulation of pelvic floor muscle, was evaluated after resting profilometry was done. The data from these tests were compared to control values.

\section{Results}

In all the subjects, the procedure of profilometry recordings was performed without any difficulty and the numerical results are shown in Table 1. Figure 3 shows a typical simultaneous recording of urethral pressure and urethral cross-sectional area profilometry on a normal male volunteer 48 yeas of age. As the balloon of the measuring probe is withdrawn to the internal meatus from the bladder, the urethral cross-sectional area begins to decrease and urethral pressure simultaneously rises. A gradual decrease in urethral cross-sectional area and a corresponding increase in urethral pressure are evident, extending from the bladder neck to the level of the external sphincter. When the balloon passes through the portion of the external sphincter, the urethral cross-sectional area decreases sharply and the urethral pres-

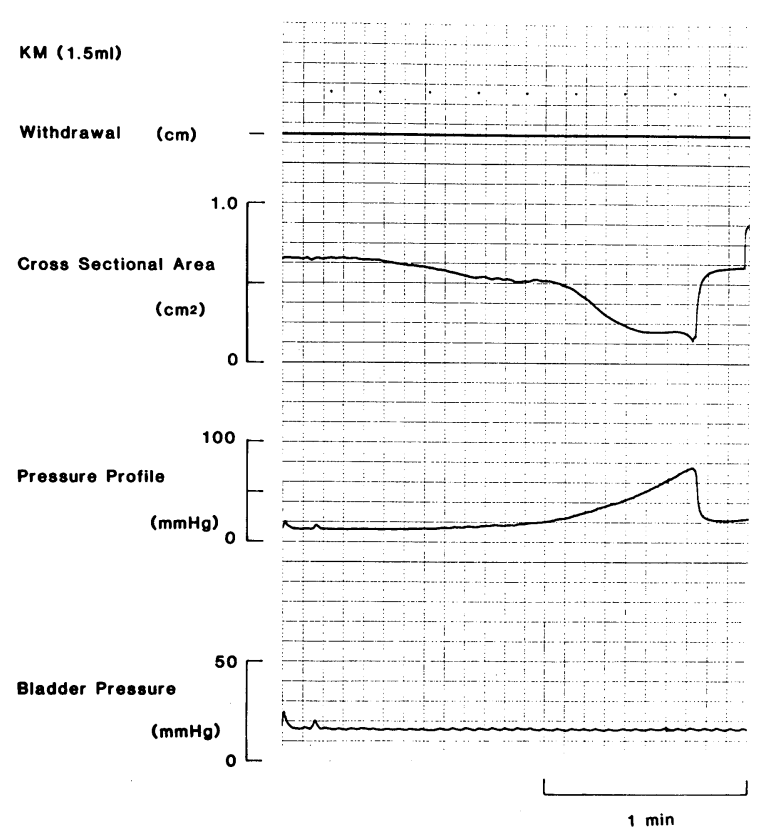

Fig. 3. Simultaneous profilometry of urethral pressure and cross sectional area at rest of normal male volunteer 48 years of age, recorded during progressive withdrawing of a catheter from the bladder to the external meatus. A gradual decrease in urethral cross sectional area and a complementary increase in urethral pressure are evident, extending from the bladder neck to the external sphincter. 
Table 1. Measurements of Healthy Male Volunteers.

\begin{tabular}{|c|c|c|c|c|c|c|c|c|}
\hline \multirow[b]{2}{*}{$\operatorname{Name}(\mathrm{n}=)$} & \multicolumn{2}{|l|}{ UCPmax* } & \multicolumn{2}{|l|}{ FUL $^{*}$} & \multicolumn{2}{|c|}{ UCSAmin * } & \multicolumn{2}{|c|}{ UCSAint* } \\
\hline & mean $\pm \mathrm{SD}(\mathrm{mmHg})$ & $\mathrm{CV}(\%)$ & mean $+\mathrm{SD}(\mathrm{cm})$ & $\mathrm{CV}(\%)$ & mean $\pm \mathrm{SD}\left(\mathrm{cm}^{2}\right)$ & $\mathrm{CV}(\%)$ & mean $\pm \mathrm{SD}\left(\mathrm{cm}^{2}\right)$ & $\mathrm{CV}(\%)$ \\
\hline K. M. $(n=4)$ & $63.2 \pm 1.3$ & 2.0 & $4.6 \pm 0.2$ & 3.7 & $0.13 \pm 0.02$ & 13.9 & $0.66 \pm 0.02$ & 3.5 \\
\hline J. S. $(n=4)$ & $61.7 \pm 2.7$ & 4.4 & $5.2 \pm 0.2$ & 3.9 & $0.15 \pm 0.01$ & 8.5 & $0.63 \pm 0.04$ & 6.0 \\
\hline K. H. $(n=3)$ & $56.3 \pm 1.5$ & 2.7 & $4.5 \pm 0.1$ & 2.6 & $0.1 \pm 0.01$ & 9.1 & $0.69 \pm 0.01$ & 2.2 \\
\hline $\operatorname{SUM}(\mathrm{n}=11)$ & $60.8 \pm 3.4$ & 5.7 & $4.8 \pm 0.4$ & 8.0 & $0.12 \pm 0.02$ & 15.7 & $0.67 \pm 0.04$ & 6.0 \\
\hline
\end{tabular}

* UCPmax : Maximum urethral closure pressure. FUL: Functional urethral length.

UCSAmin: Minimum urethral cross sectional area. UCSAint: Urethral cross sectional area the internal meatus
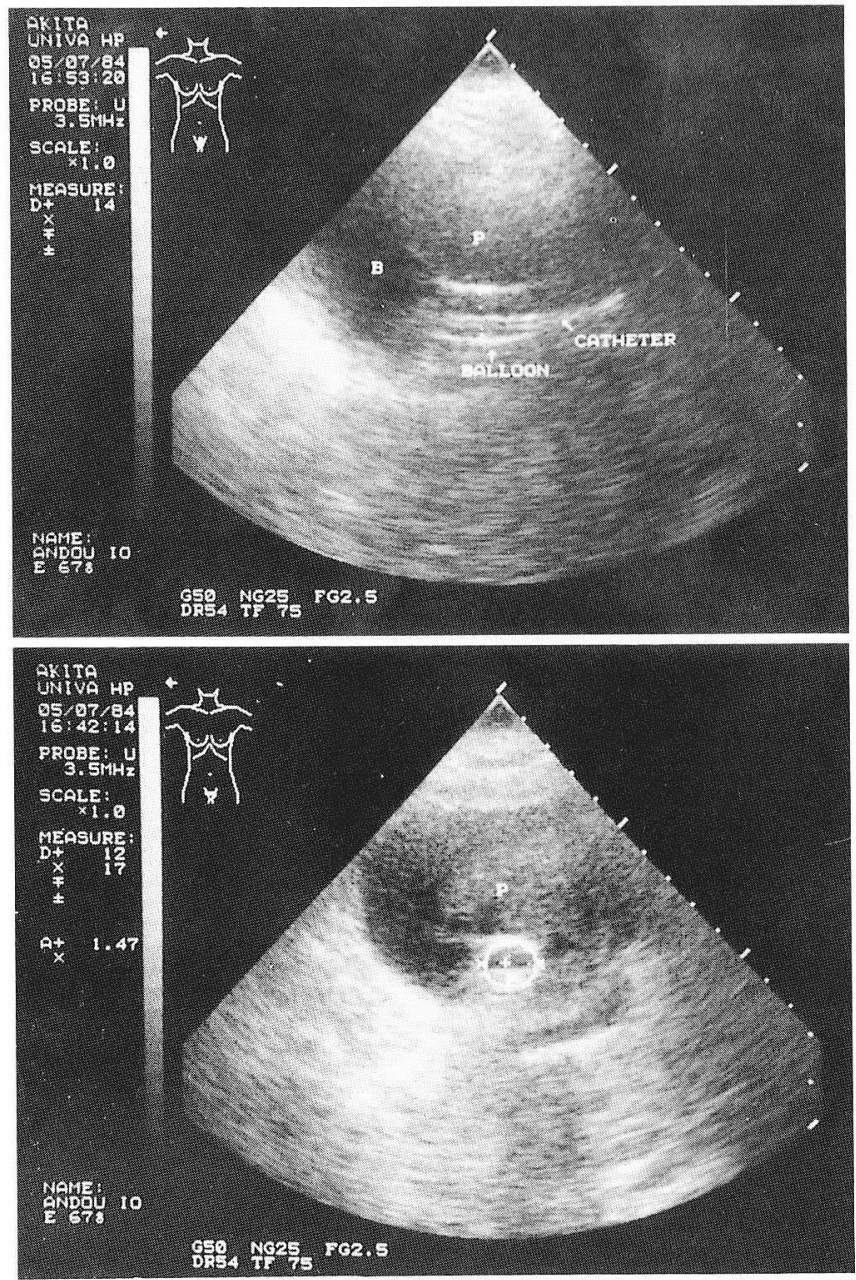

Fig. 4. Transabdominal echogram of the prostatic urethra during simultaneous profilometry of urethral pressure and cross sectional area. The echogram shows the distention of the prostatic urethra by the balloon. The cross sectional area of the balloon is $0.71 \mathrm{~cm}^{2}$, measured by the echogram (transverse length: $1.0 \mathrm{~cm}$, longitudinal length: $0.9 \mathrm{~cm}$ ) and $0.63 \mathrm{~cm}^{2}$, measured by the simultaneous profilometry of urethral pressure and cross sectional area. 
sure increases to maximum. As shown in Figure 4, the position of the balloon and also the cross-sectional area of the balloon were confirmed by transabdominal ultrasound imaging.

Analysis of the parameters obtained from the recordings of three normal male volunteers are shown in Table 1. As indicated in this table, the individual coefficients of variation of UCPmax and FUL are less than 5\%.

Figure 5 shows the urethral pressure and cross-sectional area of a patient with benign prostatic hypertrophy, estimated at 82 grams of adenoma, using ultrasound examination. As indicated in figure 6, there is elongation of FUL and UCPmax is high. Regular vascular pulse waves in the prostatic urethra were also recognized.

Figure 6 illustrates recording of urethral pressure and cross-sectional area during cough in a normal male volunteer. As indicated, the cross-sectional area of the prostatic urethra decreases, indicating physical closure of the proximal urethra. The urethral pressure at the site increased simultaneously, also indicating compression of the urethra. The extent of urethral pressure rise is shown to correspond to bladder-pressure elevation due to cough. However, in the region of the external sphincter, there is no significant cross-sectional area decrease, presumably since the maximum compression was achieved and the urethra is as closed as it could be.

Recording of urethral profilometry in a patient with incontinence after a transurethral prostatectomy is shown in Figure 7. The maximal urethral closure pressure is $48 \mathrm{mmHg}$, which is lower than expected for normal volunteers. The minimal urethral cross-sectional area in that region is approximately the same as normal values. Voluntary contraction of the perineal musculature in response to the command of "squeeze" produces a rise in pressure and

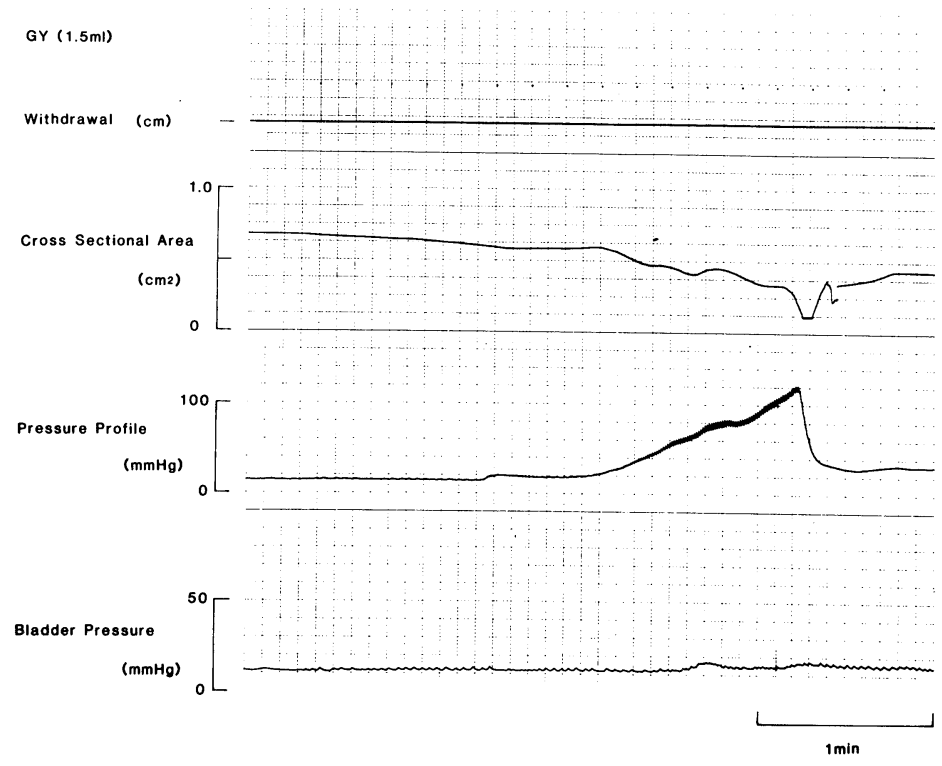

Fig. 5. Simultaneous profilometry of urethral pressure and cross sectional area at rest of patient with benign prostatic hypertrophy, 64 years of age. The elongation of functional urethral length in the proximal urethra and high value of maximal urethral closure pressure are evident. Note the regular pulse wave in prostatic urethral pressure, indicating compression of vascularity in that region. 


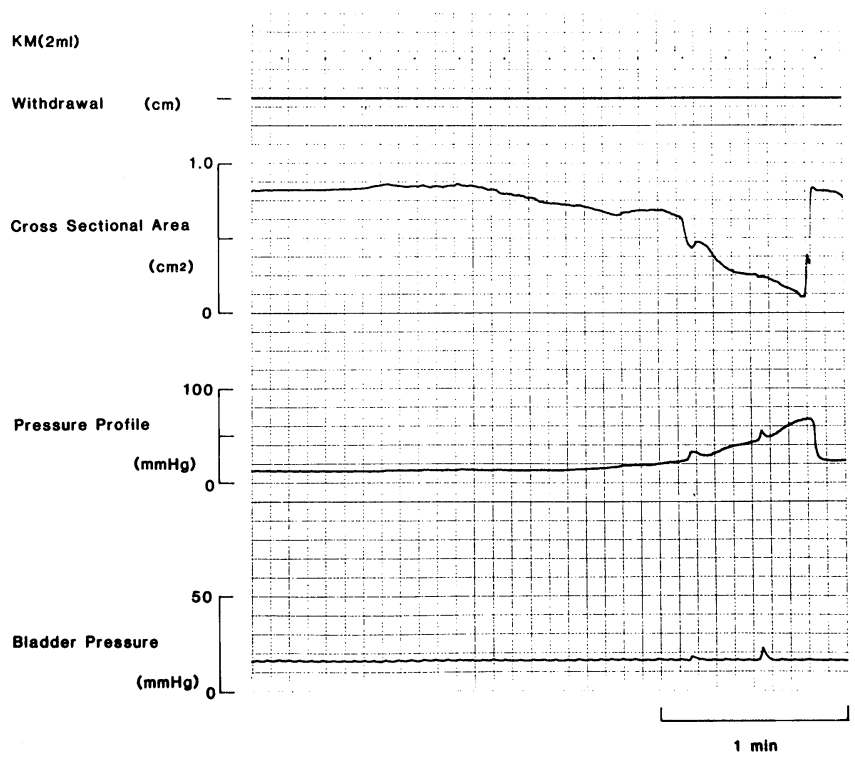

Fig. 6. Simultaneous profilometmy of urethral pressure and cross sectional area, while coughing, of normal male volunteer 48 years of age. With coughing, there is a sharp increase in bladder pressure and urethral pressure, and decrease in urethral cross sectional area.

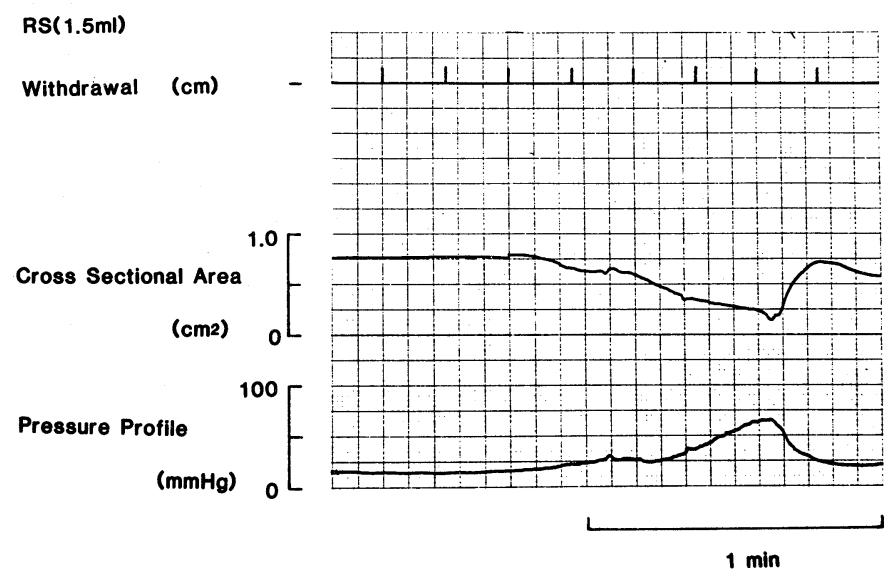

Fig. 7. Simultaneous profilometry of urethral pressure and cross sectional area at rest of patient with incontinence after transurethral resection of the prostate, 68 years of age. Maximal urethral closure pressure in $48 \mathrm{mmHg}$, which is lower than expected of normal subject.

a further decrease of cross-sectional area of the external sphincteric urethra (Figure 8).

Figure 9 illustrates the change of urethral closure pressure and cross-sectional area during field electrical stimulation of the pelvic floor provided by an anal plug in a patient with stress incontinence. As shown, electrical stimulation used alone does not produce a significant response on urethral closure. However, electrical stimulation combined with voluntary squeezing causes a greater rise of urethral pressure and a decrease in urethral cross-sectional area. 


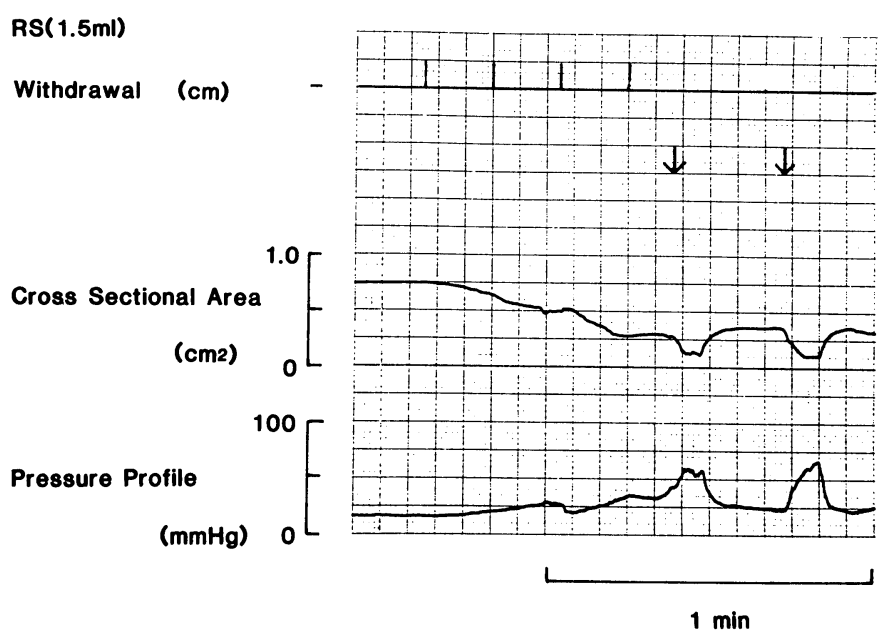

Fig. 8. Simultaneous profilometry of urethral pressure and cross sectional area with voluntary squeeze of the same patient as presented in Fig. 7. The balloon is stabilized near the external sphincter. There is a sharp increase of urethral pressure and simultaneous decrease of urethral cross sectional area in response to the command of "squeeze".

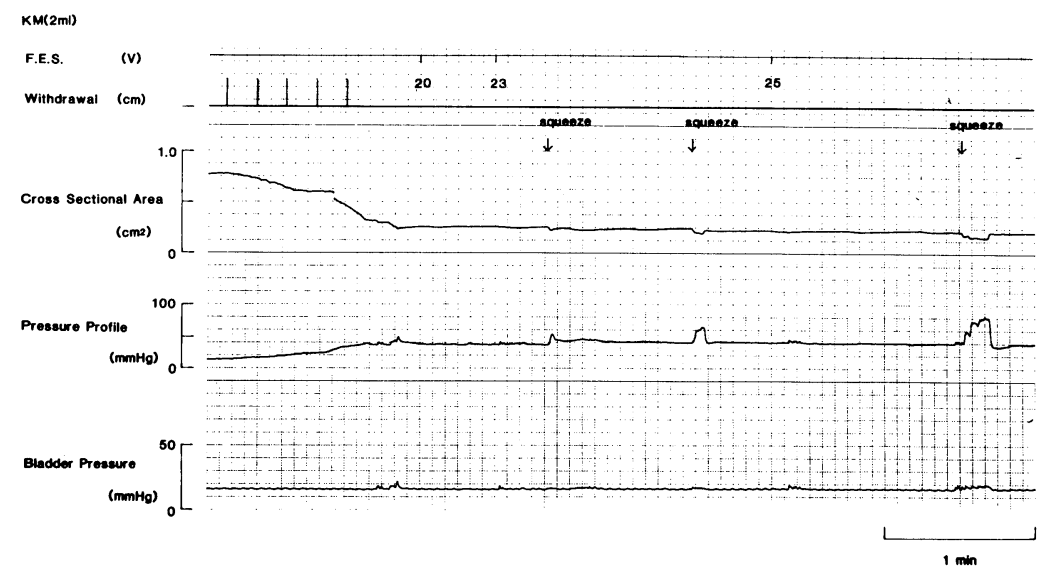

Fig. 9. Simultaneous profilometry of urethral pressure and cross sectional area at different levels of electrical stimulation of a patient with incontinence after rectal surgery. The balloon is stabilized near the external sphincter. The stimulation of $20 \mathrm{~Hz}$ in frequency and 2 msec in pulse length was applied by anal plug. Electrical stimulation combined with voluntary squeezing produces a rise of urethral pressure and decrease of the cross sectional area.

\section{Discussion}

The advantage of our new method presented here is that the simultaneous recording of both pressure and cross-sectional area of the urethra is presented immediately in graphic form. Technically, the procedure is simple and can be performed in a short time. Furthermore, use of this method determines not only the anatomical configuration but also the physiological movement of the urethra while the bladder is filling. This method is also useful in exploring how urethral closure changes when the patient assumes different posture (sitting or standing) 
or undergoes various stress (coughing or squeeze)

Since the pressure recorded using the fluid perfusion or micro-tip transducer produces data that are localized at a small site of measurement, depending on orientation of the catheter, the pattern of the urethral pressure profile is substantially influenced by the axis orientation of the catheter (van Green et al., 1984)

As the coefficient of variation in our results indicates, our new method presents a high degree of reproducibility in measuring urethral pressure, because the catheter has a relatively wide and circumferential membrane which is equally sensitive to pressure change from all directions. In that sense, it represents urethral closure more accurately. The pulse waves recorded in the prostatic urethra (Figure 5) clearly demonstrate that there is reasonable frequency response of the pressure-measuring system (Asmussen et al., 1976). In this way, it is likely that this frequency response will also be useful during stress (coughing) testing of the urethra.

The variances of the measurement of cross-sectional area in the present report are slightly higher than those of pressure measurements. This increase in variances is considered to be due to errors in the position of the cross-sectional area probe in relation to the center axis of the balloon. In addition, the larger length of the balloon is likely to be more liable to the registration of pressures originating from periurethral strictures.

Both recordings of urethral pressure and cross-sectional area respond sharply to the abdominal pressure elevation due to cough or voluntary contraction of the pelvic floor muscles following the command "Squeeze". Moreover, the recorded values of pressure and crosssectional area have quantitative significance in absolute terms of the physical parameters involved, and are not restricted to comparative values. Consequently, it seems that our new method is not only applicable in a clinical setting but may also prove useful in basic investigation of the mechanisms of relaxation and constriction of the urethra and micturition in general.

\section{References}

Asmussen, M. and Ulmsten, U. (1976). Simultaneous urethro-cystometry with a new technique. Scand. J. Urol. Nephrol., $10: 7-11$.

Brown, M. and Wicham, J.E.A. (1969). The urethral pressure profile. Br. J. Urol., 41: 211-217.

Colstrup, H., Mortensen, S.O. and Kristensen, J.K. (1983). A new method for the investigation of the closure function of the resting female urethra. J. Urol., 130: 507-511.

Enhorning, G. (1961). Simultaneous recording of the intravesical and intraurethral pressure. Acta Chir. Scand. (Suppl.), $276: 1-68$.

Harada, T. (1985). Experimental study on measurements of compliance and cross sectional area in the urethra through field gradient principle. Jap. J. Urol., 76: 360-366.

Harada, T., Kumazaki, T., Kigure, T., Etori, K. and Tsuchida, S. (1989). Effect of adrenergic agents on urethral pressure and urethral compliance measurements in dog proximal urethra. $J$. Urol., 142: 189-192.

Regnier, C.H., Susset, J.G., Gohniem, G.M. and Baincani, P. (1983). A new catheter to measure urethral compliance in females: Normal values. J. Urol. , 129: 1060-1062.

Shmidt, R.A., Witherow, R., van Gool, J. and Tanagho, E.A. (1976). Urethral pressure profilometry with membrane catheter compared with perfusion catheter system. Urol. Int., 33 : 345-351.

Simons, I. (1936). Cystometry: Studies in bladder function. 2. The sphincterometer. J. Urol., 35 : 
96-102.

Tanagho, E.A., Mayers, F.H. and Smith, D.R. (1967). Urethral resistance: Its components and implications. I-smooth muscle component. Invest. Urol., 7: 136-142.

van Geelen, J.M., Doesburg, W.H. and Martin, C.B. Jr. (1983). Female urethral pressure profile; reproducibility, axial variation and effects of low dose oral contraceptives. J. Urol. 131 : 394-398. 